\title{
Compact Adaptive Optics Scanning Laser Ophthalmoscope with Phase Plates
}

\author{
Gopal Swamy Jayabalan, Ralf Kessler, \\ Jörg Fischer, and Josef F. Bille
}

\subsection{Introduction}

One of the major concerns in ophthalmology is to preserve vision since even a minor loss in visual acuity can severely impact the quality of life. Since the invention of the first ophthalmoscope in 1851 by Hermann von Helmholtz, fundus imaging has become an essential part of the retinal examination for diagnostic and monitoring purposes. Lately, many advanced retinal imaging techniques have emerged to provide new insights into the pathogenesis of retinal diseases. A broad range of clinical instruments like slit lamp, fundus camera, direct and indirect handheld ophthalmoscopes, confocal scanning laser ophthalmoscope (cSLO), optic nerve head analyzer, ultrasonography and optical coherence tomography (OCT) has been widely used in clinics for early diagnosis and monitoring retinal disease progression. However, these instruments do not help to identify the early stages of retinal diseases since the pathogenesis begins at a cel-

G. S. Jayabalan $(\bowtie) \cdot$ J. F. Bille

Heidelberg Engineering GmbH,

Heidelberg, Germany

University of Heidelberg, Heidelberg, Germany

R. Kessler · J. Fischer

Heidelberg Engineering GmbH,

Heidelberg, Germany lular level, and visualization of changes at a microscopic level is required [1]. Therefore, a major effort in developing new retinal imaging techniques at a cellular level is essential.

Retinal imaging at a cellular level is challenging since the instrument should have a lateral resolution approaching the cell size to resolve neighboring cells within the same focal plane, combined with penetration through absorbers and scatter in the eye, optical sectioning, speed, sensitivity, and contrast generation. Likewise, to visualize individual cells the camera should have sufficient resolution and contrast. With handheld ophthalmoscopes and fundus cameras, the gross anatomical features of the retina over large areas can be observed, but these instruments do not provide clinically relevant information at a cellular level. The cSLO and OCT have a better effective resolution than the fundus camera and have been widely implemented for various clinical applications including detection of the biomarkers of diabetic retinopathy, AMD, and glaucoma. Regardless of these technological advancements, imaging of the retina at a cellular level was limited by the optical aberrations which limit the lateral resolution. The human eye is not a perfect optical system and ocular aberrations impair the vision as well as the retinal image quality. Although the defocus and astigmatism can be corrected by spectacles, the idea of correcting the higher-order aberrations 
in the human eye by customized contact lenses was proposed in the early 1960s by Smirnov. Later in 1997, Liang et al. successfully corrected the higher-order aberrations providing normal eyes with supernormal optical quality allowing imaging of the retina at a microscopic scale [2]. Since then, adaptive optics has been implemented by numerous research groups for high-resolution imaging of the retina. The aberrations can be compensated by integrating the adaptive optics which can also enable visualization of cone photoreceptors, rod photoreceptors, and leucocytes. In vivo retinal imaging of these structures helps to non-invasively monitor the retinal functions, the progression of retinal diseases and efficacy of therapies at a microscopic spatial scale [3].

Adaptive optics has been combined with techniques including scanning laser ophthalmoscopy, funduscopy, and OCT. In general, the adaptive optics systems are composed of two main components: a wavefront sensor and a deformable mirror. The wavefront sensor measures the aberrations induced by the optical system and eye; a deformable mirror corrects the aberrations by physically changing the surface shape to match the measured aberration. Thus, adaptive optics enables an aberration-free or a diffraction-limited system [4]. However, the cost and complexity of adaptive optics ophthalmoscopes currently obstruct its clinical use. To overcome this factor, a compact non-adaptive optics ophthalmoscope at low cost has been developed by researchers to visualize cone photoreceptors and nerve fiber bundles. Yet, the foveal rod and cone photoreceptors can be visualized only with adaptive optics ophthalmoscopes.

Even though the image quality and resolution have improved with non-adaptive optics ophthalmoscopes, the cone photoreceptors can be visualized only in healthy eyes with minimum ocular aberrations and smaller pupil size [5]. The ocular aberrations are lesser in smaller pupil size and the aberrations increase with a rise of the pupil size. For small pupil sizes (2-3 mm), diffraction dominates the Point spread function (PSF) and hence the quality of the retinal imaging, while for large pupils the major source of degradation in the PSF and retinal image quality are due to the aberra- tions. Also, the efficacy of an ophthalmic scanning laser imaging systems is dependent on the effective spot size of a laser system. Therefore, it is vital that the system's laser beam converge to diffraction-limited focal spot. The eye itself influences the imaging beam by significant optical and phase aberrations, and these aberrations have to be compensated, either removed or minimized, for diffraction-limited imaging. In principle, the diffraction limit can be achieved by establishing a flat wavefront of the imaging beam. The flat wavefront can be achieved by measuring the aberrations and producing a phase plate on the basis of the resultant measurements to compensate these aberrations. The wavefront detection technology has been widely used for refractive surgeries and allows surgeons to customize the procedure for each eye [6]. Also, the same technique is combined with the aberration correction unit in the adaptive optics system to improve the retinal image quality, see Chap. 16 for adaptive optics techniques. Likewise, it is also logical to use a "phase plate" to compensate the wavefront aberrations to facilitate high-resolution and contrast of images from aberrated eyes. This will improve the overall efficacy of resolution and contrast of scanning laser ophthalmoscopes [7]. The improvement in contrast and resolution of retinal images allows for a direct observation of retinal microstructures and analysis of their integrity and pathological abnormalities. Thus, a compact adaptive optics can be achieved with minimum cost and less complexity and can be easily incorporated in the prevailing cSLOs in clinics.

In this chapter, the design and development of a phase plate for cSLO is described to compensate the higher-order aberrations of the eye and to improve the contrast of retinal imaging.

\subsection{Wavefront Aberrations}

A typical optical imaging system consists of an object plane, an optical system and an image plane as shown in Fig. 18.1. A wavefront is a surface over which an optical disturbance has a constant phase, and is always perpendicular to the rays. The wavefront aberration is the opti- 
Fig. 18.1 Optical imaging system with right-handed coordinates

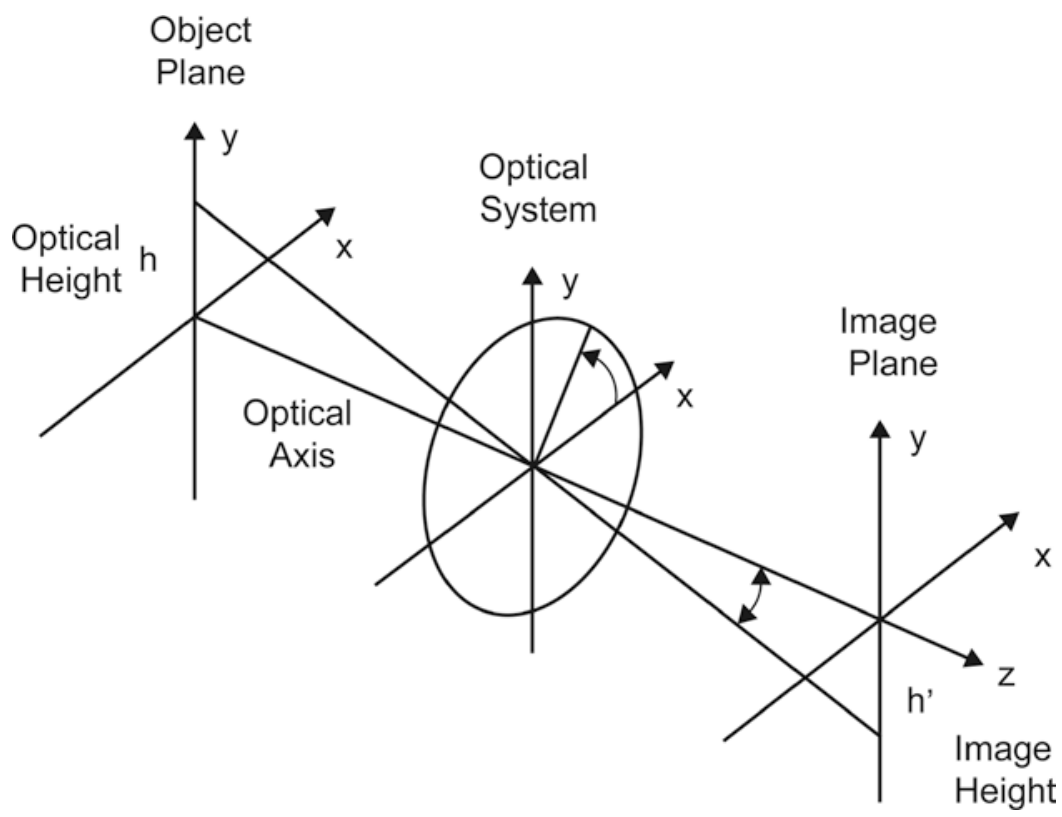

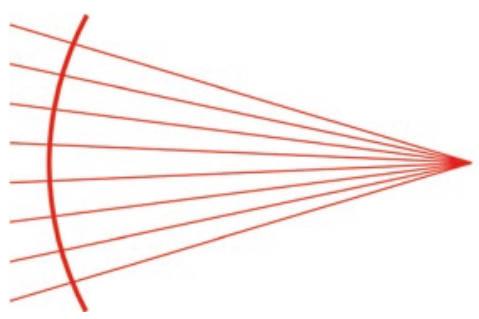
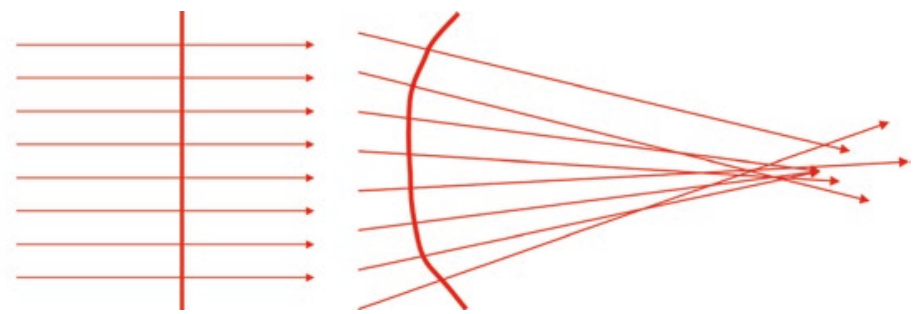

Fig. 18.2 Wavefront aberrations. Ideal spherical wavefront (left image), ideal planar wavefront (middle image), aberrated wavefront (right image)

cal path length difference along rays between the actual wavefront and the ideal wavefront at the exit pupil. The shape of the wavefront can be either spherical or planar in an aberration-free optical system (Fig. 18.2). In ophthalmology, the wavefront aberrations are most commonly expressed with Zernike polynomials and this is described in Sect. 18.2.2.1.

\subsubsection{Optical Aberrations of Human Eye}

The eye as an optical system focuses the entering light rays on the retina. The cornea is a transparent structure in front of the eye that helps to focus the incoming light. The image-forming light is further focused by the lens on the retina, and these optical signals are converted to neural signals at the retina and enable individuals to see the world. Any imperfections in focusing the light on the retina will cause the light rays to deviate, and these deviations are referred to as optical or wavefront aberrations. These aberrations will lead to blurred images and decrease in visual performance.

Wavefront aberrations are of two types:

- Lower-order aberrations

- Higher-order aberrations

Lower-order aberrations are myopia, hyperopia, and astigmatism which can be corrected with glasses, contact lenses or refractive surgery. These aberrations make up about $85 \%$ of aberrations in the eye. The other not visually 
significant lower-order aberrations are known as first-order aberrations, such as prisms and zeroorder aberrations (piston).

There are numerous higher-order aberrations, of which coma, trefoil, and spherical aberration are of clinical interests in ophthalmology. Coma is the distortion in the image formation and occurs when the light rays entering the optical systems are not parallel to the optic axis. Spherical aberration is an imaging imperfection that occurs when the light rays from the edges of a lens or mirror focus at a shorter distance than the light rays from the center. Higher-order aberrations are more complex than the lowerorder aberrations, and these aberrations produce vision errors such as difficulty when seeing at night, glare, halos, blurring, starburst patterns or double vision. With the latest technological advancement, these aberrations can be measured and diagnosed [8].

\subsubsection{Quantitative Expression of Ocular Aberrations}

\subsubsection{Zernike Polynomials}

The most common method to classify the shapes of aberration maps is to consider each map as the sum of fundamental shapes or basic functions. In ophthalmology, the wavefront aberrations are expressed using a series of Zernike polynomials (Table 18.1). The benefit of expressing the aberrations in Zernike's polynomials is that the polynomials are independent to each other, and the coefficient gives the wavefront errors.

A Zernike polynomial is a complete set of functions orthogonal over the unit circle (Fig. 18.3) and parameterized by a dimensionless radial parameter $\rho$ and a dimensionless meridional parameter $\theta$. It is designated by a non-negative radial integer index $n$ and a signed meridional index $m$. Each Zernike polynomial is the product of three terms, a normalization term, radial term and a meridional term. It is given by the following equation.

$$
\begin{aligned}
Z_{n}^{m}(\rho, \theta)= & N_{n}^{m} R_{n}^{|m|}(\rho) \cos (m \theta) \\
& \text { for } \mathrm{m} \geq 0,0 \leq \rho \leq 1,0 \leq \theta \leq 2 \pi \\
Z_{n}^{-m}(\rho, \theta)= & -N_{n}^{m} R_{n}^{|m|}(\rho) \sin (m \theta) \\
& \text { for } \mathrm{m}<0,0 \leq \rho \leq 1,0 \leq \theta \leq 2 \pi
\end{aligned}
$$

for a given $n: m$ can only take on values $-n$, $-n+2,-n+4, \ldots . n$.

$N_{n}^{m}$ is the normalization factor and is given by

$$
\begin{aligned}
& N_{n}^{m}=\sqrt{\frac{2(n+1)}{1+\delta_{m 0}}} \delta_{m 0}=1 \text { for } \mathrm{m}=0, \\
& \delta_{m 0}=0 \text { for } m \neq 0
\end{aligned}
$$

$R_{n}^{|m|}(\rho)$ is the radial polynomial and is given by

$$
\begin{aligned}
& R_{n}^{|m|}(\rho)=\sum_{s=0}^{(n-|m|) / 2}(-1)^{s}(n-s) ! \\
& \frac{s ![0.5(n+|m|)-s] ![0.5(n-|m|)-s] !}{\rho^{n-2 s}}
\end{aligned}
$$

\subsubsection{Standard Wavefront Error Description}

The wavefront error of an eye is the optical path length, i.e. the product of the geometric length (physical distance the light travels) and the refractive index of the medium, between a plane wavefront in the eye's entrance pupil and the wavefront of light exiting the eye from a point source on the retina. It is specified as a function of $(\mathrm{x}, \mathrm{y})$ or $(\rho, \theta)$ coordinates of the entrance pupil. Wavefront errors are measured in an axial direction from the pupil plane towards the wavefront. By convention, the wavefront error is set to zero at the pupil center by subtracting the central value from values at all other pupil locations.

The wavefront described using the Zernike polynomial functions as shown below.

$$
W(\rho, \theta)=\sum_{n, m} c_{n}^{m} Z_{n}^{m}(\rho, \theta)
$$

Where c denotes the Zernike amplitudes or coefficients and $\mathrm{Z}$ the polynomials. 
Table 18.1 Zernike polynomials up to fourth order

\begin{tabular}{|c|c|c|c|}
\hline Symbol & Polar form & Common name & Plots \\
\hline$Z_{0}^{0}$ & 1 & Piston & \\
\hline$Z_{1}^{-1}$ & $2 \rho \sin (\theta)$ & Vertical tilt & \\
\hline$Z_{1}^{1}$ & $2 \rho \cos (\theta)$ & Horizontal tilt & \\
\hline$Z_{2}^{-2}$ & $\sqrt{6} \rho^{2} \sin (2 \theta)$ & Oblique astigmatism & \\
\hline$Z_{2}^{0}$ & $\sqrt{3}\left(2 \rho^{2}-1\right)$ & Defocus & \\
\hline$Z_{2}^{2}$ & $\sqrt{6} \rho^{2} \cos (2 \theta)$ & Vertical astigmatism & \\
\hline$Z_{3}^{-3}$ & $\sqrt{8} \rho^{3} \sin (3 \theta)$ & Oblique trefoil & \\
\hline$Z_{3}^{-1}$ & $\sqrt{8}\left(3 \rho^{3}-2 \rho\right) \sin (\theta)$ & Vertical coma & \\
\hline$Z_{3}^{1}$ & $\sqrt{8}\left(3 \rho^{3}-2 \rho\right) \cos (\theta)$ & Horizontal coma & \\
\hline$Z_{3}^{3}$ & $\sqrt{8} \rho^{3} \cos (3 \theta)$ & Horizontal trefoil & \\
\hline$Z_{4}^{-4}$ & $\sqrt{10} \rho^{4} \sin (4 \theta)$ & Oblique quadrafoil & \\
\hline$Z_{4}^{-2}$ & $\sqrt{10}\left(4 \rho^{4}-3 \rho^{2}\right) \sin (2 \theta)$ & Oblique secondary astigmatism & \\
\hline$Z_{4}^{0}$ & $\sqrt{5}\left(6 \rho^{4}-6 \rho^{2}+1\right)$ & Primary spherical & \\
\hline$Z_{4}^{2}$ & $\sqrt{10}\left(4 \rho^{4}-3 \rho^{2}\right) \cos (2 \theta)$ & Vertical secondary astigmatism & \\
\hline$Z_{4}^{4}$ & $\sqrt{10} \rho^{4} \cos (4 \theta)$ & Horizontal quadrafoil & \\
\hline
\end{tabular}


Right Eye (OD)
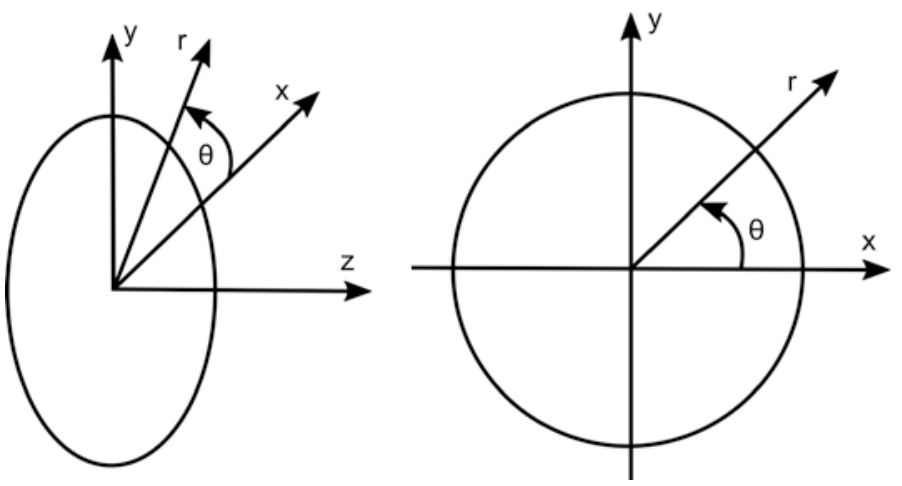

Left Eye (OS)

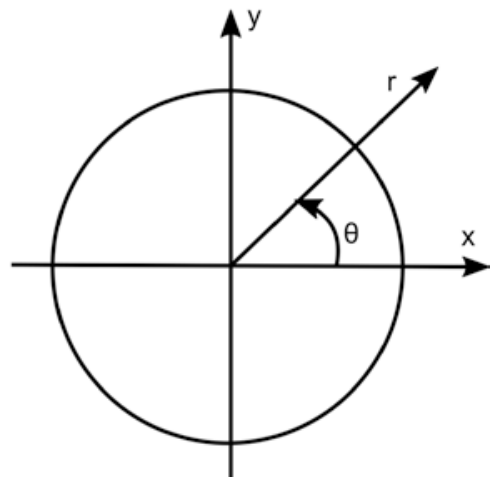

Fig. 18.3 Ophthalmic coordinate system

\subsubsection{Root Mean Square Wavefront Error}

The quantitative comparisons between different eyes and conditions are expressed as root mean square (RMS). The RMS wavefront error for the human eye is computed as the square root of the variance of wavefront error function. Piston and tilt are usually excluded from the calculation since they correspond to lateral displacements of the image rather than image degradation. RMS error is defined by the following equation.

$$
R M S=\sqrt{\frac{\iint_{\text {pupil }}[W(x, y)-\bar{W}]^{2} d x d y}{A}}
$$

Where A is the area of the pupil and $\bar{W}$ is the mean wavefront optical path difference.

If the wavefront function is expressed in terms of normalized Zernike coefficients, the RMS value is equal to the square root of the sum of the squares of the coefficients with radial indices $n \geq 2$.

$$
R M S=\sqrt{\sum_{n \geq 2, a l l m}\left(c_{n}^{m}\right)^{2}}
$$

\subsubsection{Point Spread Function}

The aberration of the human eye negatively impacts the retinal image quality. To characterize the effects of aberrations, the Fourier optics has been introduced in ophthalmology. One of those techniques is the PSF, which defines the propagation of electromagnetic radiation or other imaging waves from a point source or point object.

In incoherent imaging systems such as fluorescent microscopes, telescopes or optical microscopes, the image formation process is linear and described by linear system theory. The process is usually formulated by a convolution equation.

$$
\text { Image }=P S F \otimes \text { Object }
$$

Image: image generated by the optical system, PSF: point spread function of the optical system,

Object: object,

$\otimes:$ convolution operator.

Figure 18.4 shows the image formation process of the optical system. The final image created by the system is the convolution of the object which is going to be imaged with the point spread function of the system. Due to the imperfection of the optical system, the image is normally blurred.

The optical properties of the eye can be characterized by the wavefront error function, which can be described by a series of Zernike polynomials. The image of a point object formed by the optical system is the point spread function or impulse response. It is defined as

$$
\operatorname{PSF}(x, y)=\frac{1}{\lambda^{2} d^{2} A_{p}}\left\|F T\left\{p(x, y) \cdot e^{-i \frac{2 \pi}{\lambda} W(x, y)}\right\}\right\|^{2}
$$



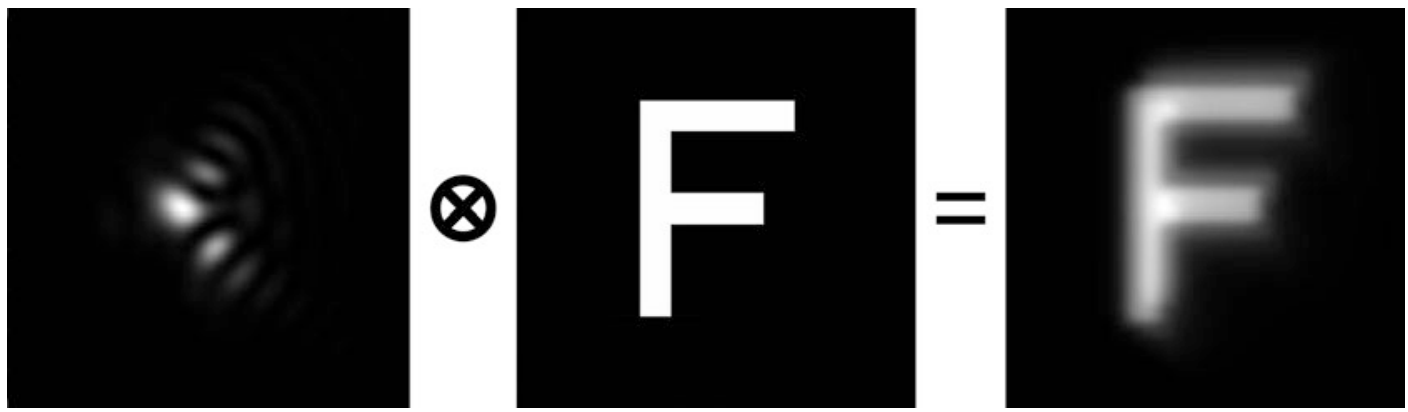

Fig. 18.4 Image formation process. The final image generated by the optical system can be described as a mathematical convolution of the object with the PSF of the optical system

FT: Fourier transform operator.

D: distance from the exit pupil to image.

$A_{p}$ : area of the exit pupil.

$P(x, y)$ : defines the shape, size and the transmission of the exit pupil.

$e^{-i \frac{2 \pi}{\lambda} W(x, y)}$ accounts for phase deviations of wavefront from a reference sphere.

$\mathrm{W}(\mathrm{x}, \mathrm{y})$ : wavefront aberrations function at the exit pupil.

It is also possible to calculate the modulation transfer function (MTF) of the human eye, which is the modulus of the optical transfer function (OTF). MTF is normally used to characterize the resolution and performance of an imaging system and is also known as spatial frequency response. The mathematical formulas are given by the following equation:

$$
\begin{gathered}
\operatorname{OTF}\left(s_{x}, s_{y}\right)=\frac{F T\{P S F\}}{F T\{P S F\} \mid s_{x}=0, s_{y}=0} \\
\operatorname{MTF}\left(s_{x}, s_{y}\right)=\left\|\operatorname{OTF}\left(s_{x}, s_{y}\right)\right\|
\end{gathered}
$$

$\left(s_{x}, s_{y}\right)$ are in units of cycles per radian.

\subsubsection{Strehl Ratio}

The Strehl ratio is a measure of the effect of aberrations in reducing the maximum or peak value of the PSF. The definition of the Strehl ratio is the ratio of the observed peak intensity at the detection plane of a telescope or other imaging systems from a point source compared to the theoretical maximum peak intensity of a perfect imaging system working at the diffraction limit. The Strehl calculation is based on a complex mathematics, and a simple empirical expression gives a very close approximation of the Strehl ratio in terms of the RMS wavefront error:

$$
\text { Strehl ratio } \cong e^{-(2 \pi \sigma)^{2}}
$$

where $\sigma$ is the root-mean-square deviation of the wavefront measured in wavelengths.

\subsubsection{Assessment of Ocular Aberrations}

Higher-order aberrations can be measured using a wavefront sensor, and Hartmann-Shack sensors have been widely used in ophthalmology to measure the monochromatic aberrations of the eye. The efficacy of this technique was first demonstrated by Junzhong Liang during his doctoral thesis at the University of Heidelberg under the supervision of Prof. Dr. Josef Bille [10]. The aberrometer measures the distortion of a light wave passing through the optics of the eye. These sensors do not measure the light scatter, chromatic aberrations or diffraction phenomena, and their effects on vision have to be assessed by other technologies. The principle of wavefront aberrometer is explained in detail in Chap. 16. 


\subsection{Experimental Setup}

\subsubsection{Confocal Scanning Laser Ophthalmoscope}

For the preliminary study, the cSLO (SPECTRALIS, Heidelberg Engineering $\mathrm{GmbH}$, Heidelberg, Germany) with the highmagnification objective lens was used. cSLO is a non-invasive imaging technique that scans the retina with a laser beam enabling high-resolution retinal imaging. The schematic of cSLO is shown in Fig. 18.5. The emitted fluorescent light (green line Fig. 18.5) from the retina can be captured using a detector and the out of focus (red line Fig. 18.5) can be eliminated by a confocal pinhole in front of the detector. With SPECTRALIS, the depth of focus can be adjusted manually and deeper tissue structures can be visualized. Threedimensional images can be generated with the focal plane adjustment as well (see Chap. 2 for more details).

The SPECTRALIS is an indispensable instrument in the field of ophthalmology combining cSLO and high-resolution OCT. It has been widely used to diagnose various retinal diseases. SPECTRALIS is an expandable diagnostic imaging platform and adds value to imaging as it can be used for conventional fundus imaging to ultra-widefield retinal imaging by simply changing the objective lenses. High-contrast fundus images can be acquired with a $30^{\circ}$ field of view (FOV) using a standard objective lens (Fig. 18.7, left image). The widefield images of the fundus can be achieved with an additional objective lens allowing for a $55^{\circ} \mathrm{FOV}$ capturing the macula, the optic nerve head and areas beyond the vessels arcades in a single image (Fig. 18.6, left image). The widefield imaging facilitates a comprehensive diagnostics beyond the conventional fundus imaging. Added, the ultra-widefield objective lens can capture an extremely wide FOV of $102^{\circ}$ with evenly illuminated, high-contrast images even in the periphery (Fig. 18.6, right image). Thus, with a single imaging platform images of different FOV can be acquired using SPECTRALIS simply by changing the objective lenses.

Similarly, the Heidelberg Engineering has developed a new lens for high-magnification retinal imaging with $8^{\circ}$ and $4^{\circ} \mathrm{FOV}$. The highmagnification objective lens is an add-on objective lens that replaces the standard objective lens to acquire a smaller FOV for higher magnification (Figs. 18.7, 18.8, 18.9, and 18.10) The pixel densities are improved in both $\mathrm{X}$ and $\mathrm{Y}$ directions with the high-magnification objective lens compared to the standard objective lens $\left(30^{\circ} \mathrm{FOV}\right)$. The digital image readout for the high-resolution mode with high-magnification objective lens for $8^{\circ}$ and $4^{\circ} \mathrm{FOV}$ is $1536 \times 1536$ and $768 \times 768$ pixels. Currently, the $4^{\circ} \mathrm{FOV}$ is possible only with the in-house test device for preliminary studies, and if the results are adequate and guarantee a future-oriented solution, the firmware will be updated with $4^{\circ} \mathrm{FOV}$ in a later release of SPECTRALIS with a high-
Fig. 18.5 Schematic diagram of cSLO

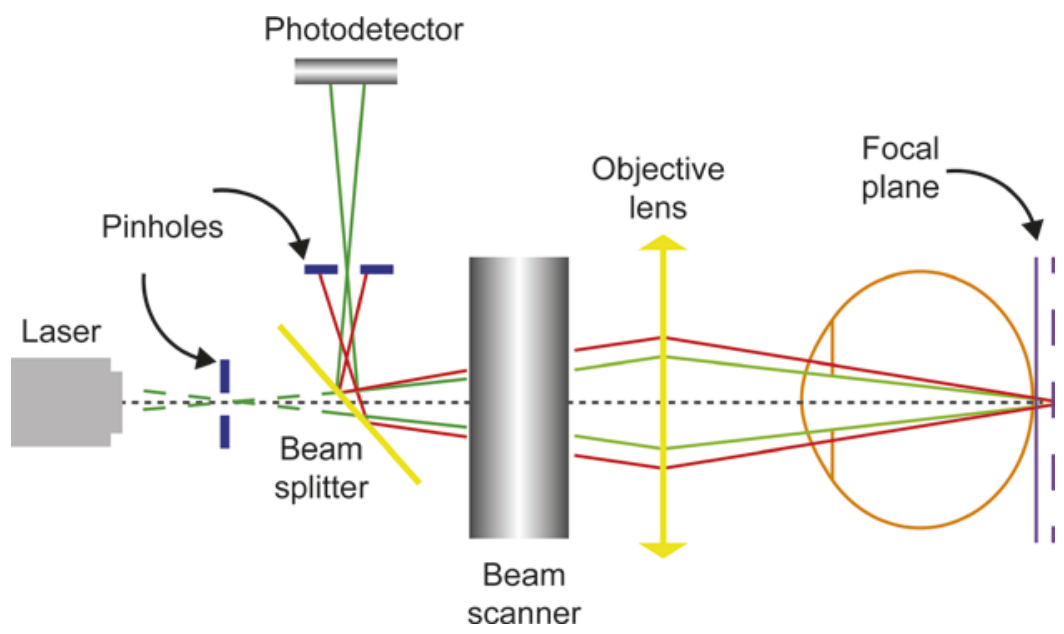



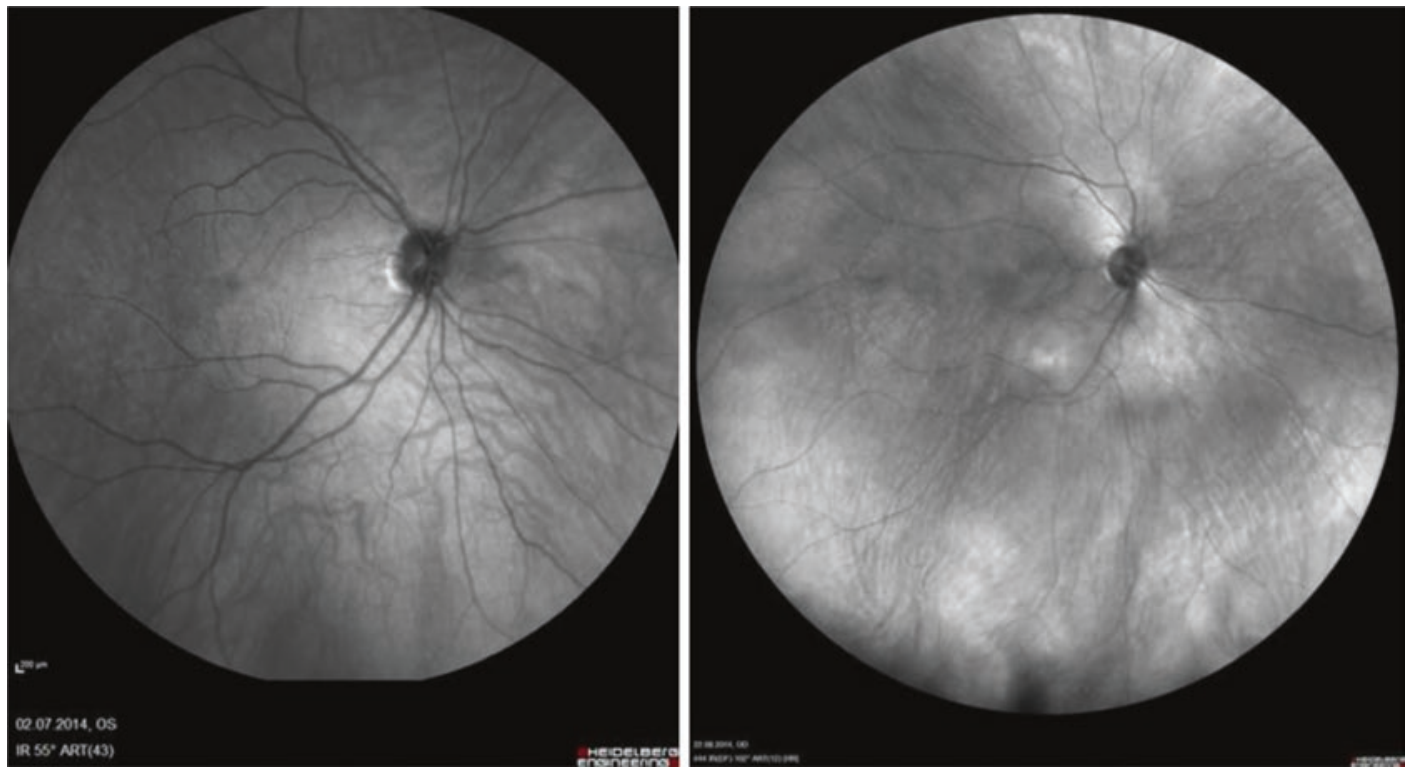

Fig. 18.6 SPECTRALIS widefield (left image) and ultra-widefield (right image) retinal image of the right eye

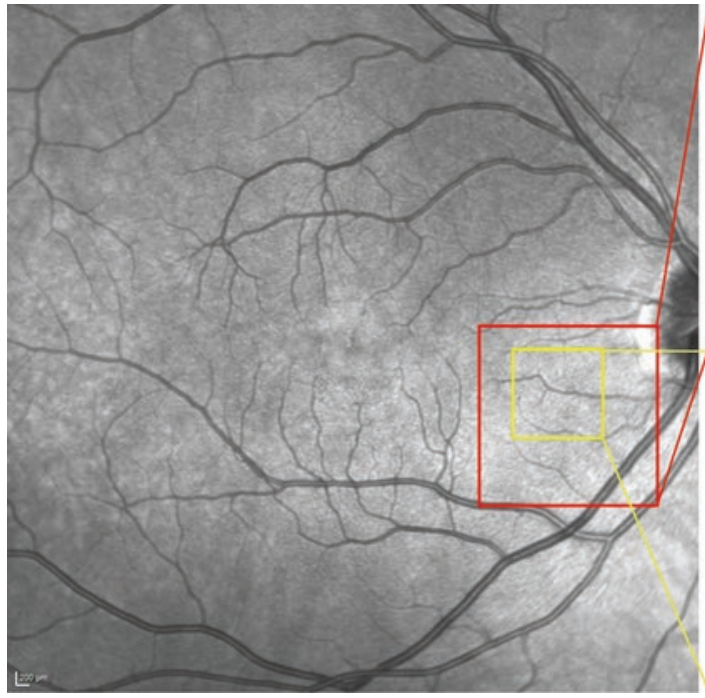

Fig. 18.7 SPECTRALIS standard infrared reflectance image $\left(30^{\circ}\right.$ FOV-left image) of the right eye. Highmagnification retinal images with $8^{\circ}$ (red box) and $4^{\circ}$

magnification objective. For laser safety, an additional blocking filter is integrated into the objective mount. This filter strongly reduces the blue laser $(486 \mathrm{~nm})$. The infrared laser $(810 \mathrm{~nm})$, indocyanine green laser $(786 \mathrm{~nm})$ and the green laser $(518 \mathrm{~nm})$ can be used for examination with
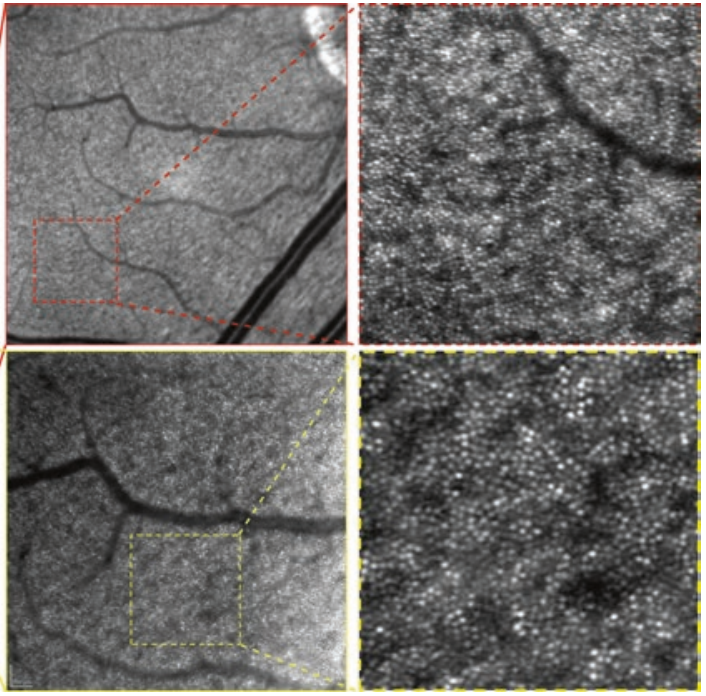

(yellow box) FOV. Zoom-in images of $8^{\circ}$ (red dotted line box) and $4^{\circ}$ (yellow dotted line box) FOV showing cone photoreceptors

a high-magnification objective lens in the reflectance mode and in the angiography mode. The cone photoreceptors can be visualized with the high-magnification objective lens in subjects with less ocular aberrations even without the use of adaptive optics. 


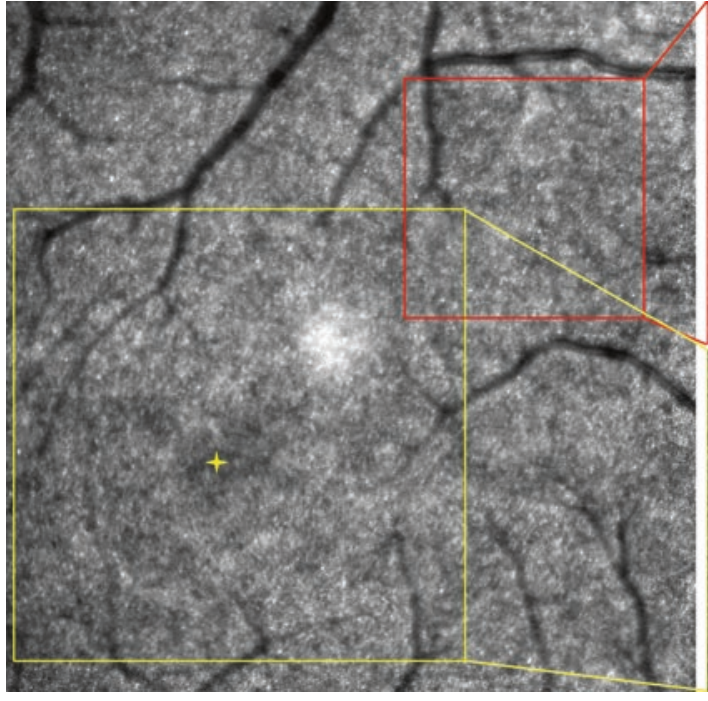

Fig. 18.8 SPECTRALIS image of the fovea (4-point star) with $8^{\circ}$ FOV (left image) showing cone photoreceptors at retinal eccentricities. Zoom-in images show-
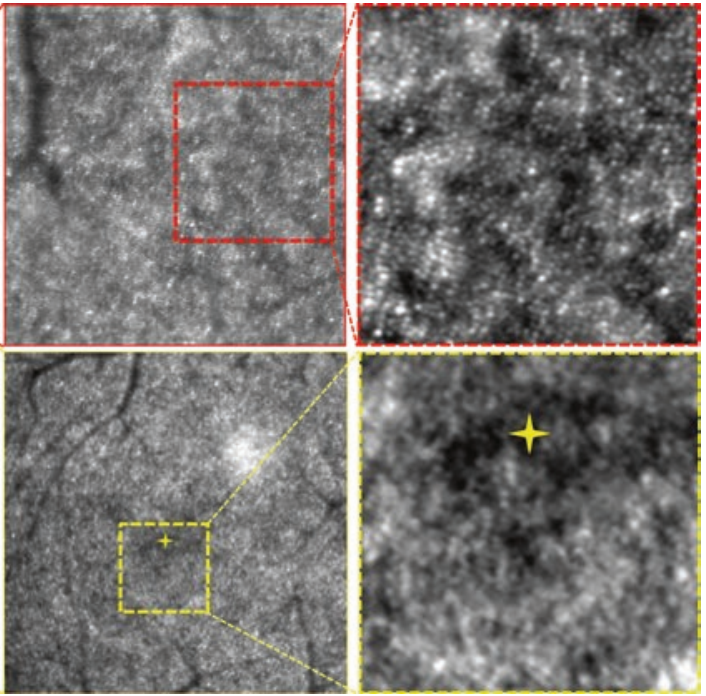

ing cones photoreceptors at the retinal eccentricities (red boxes) and no photoreceptors at the fovea (yellow boxes)
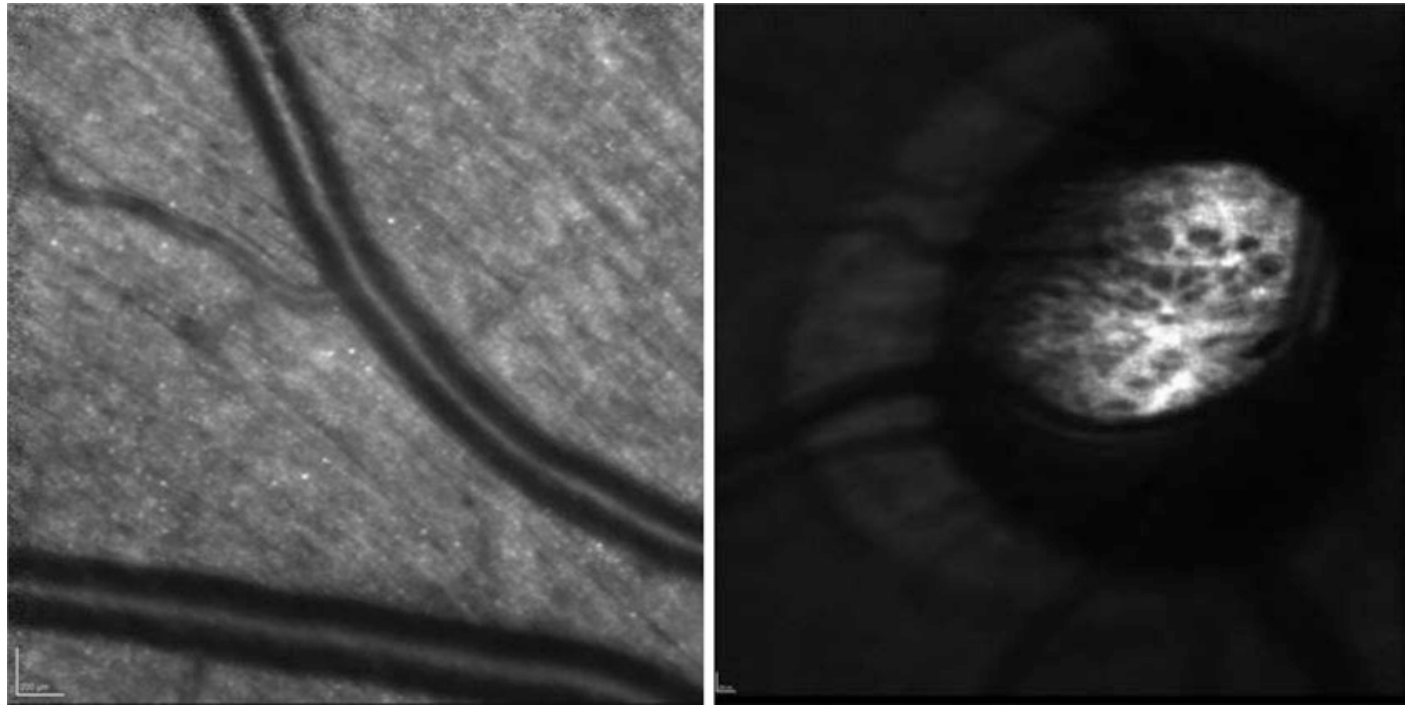

Fig. 18.9 SPECTRALIS with high-magnification objective lens showing nerve fiber bundles (left image) and lamina cribrosa (right image)

\subsubsection{Measurement of Ocular Aberrations}

Many commercially available aberrometers can be used to measure the ocular aberrations of an eye to produce a customized phase plate for each individual. For this study, we used a commercially available aberrometer iDesign ${ }^{\mathrm{TM}}$ from
Abbott Medical Optics which combines aberrometry and corneal topography measurements (Fig. 18.11). The wavefront sensor component in this instrument is a Hartmann-Shack wavefront sensing type. With the measured ocular aberrations, a customized phase plate for each individual was produced. The ocular aberrations measurements were performed at the eye clinic in 

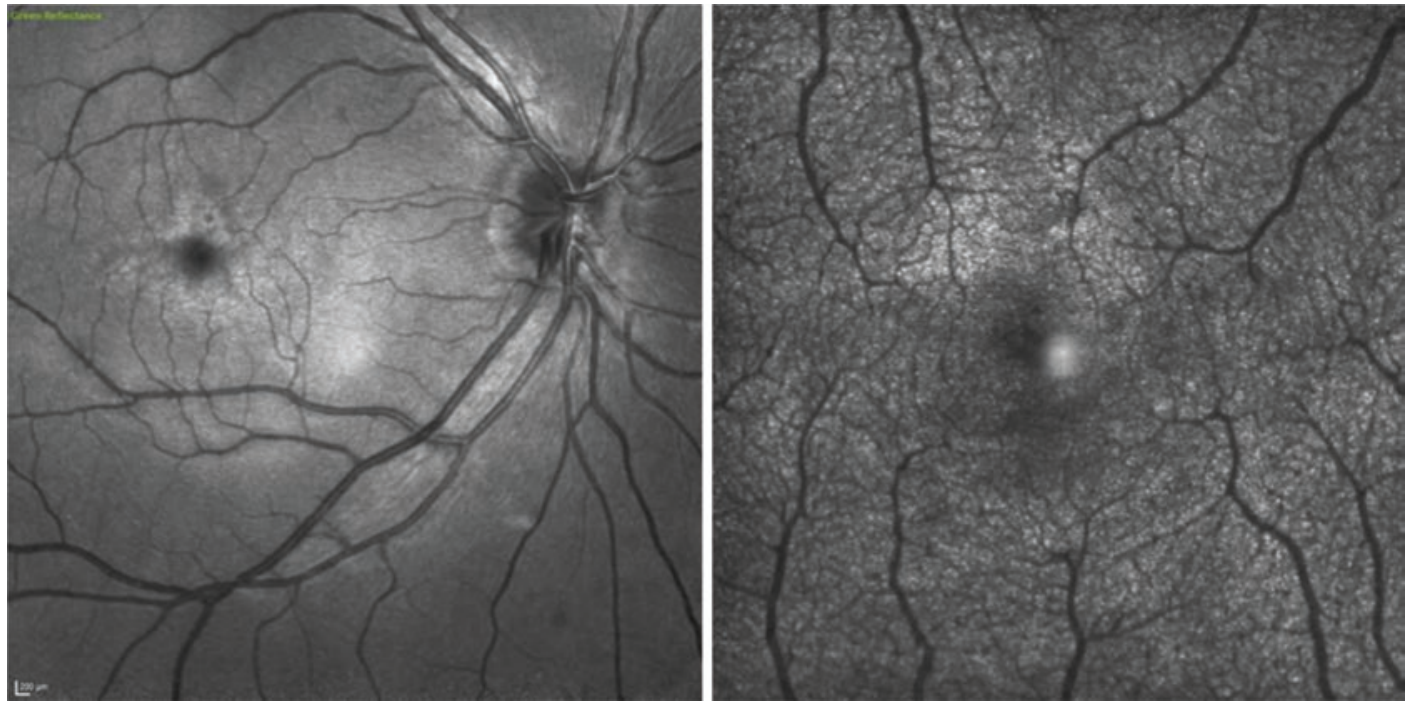

Fig. 18.10 Green light retinal image with standard $\left(30^{\circ}\right.$ FOV—left image $)$ and high-magnification $\left(8^{\circ}\right.$ FOV—right image) objective lenses

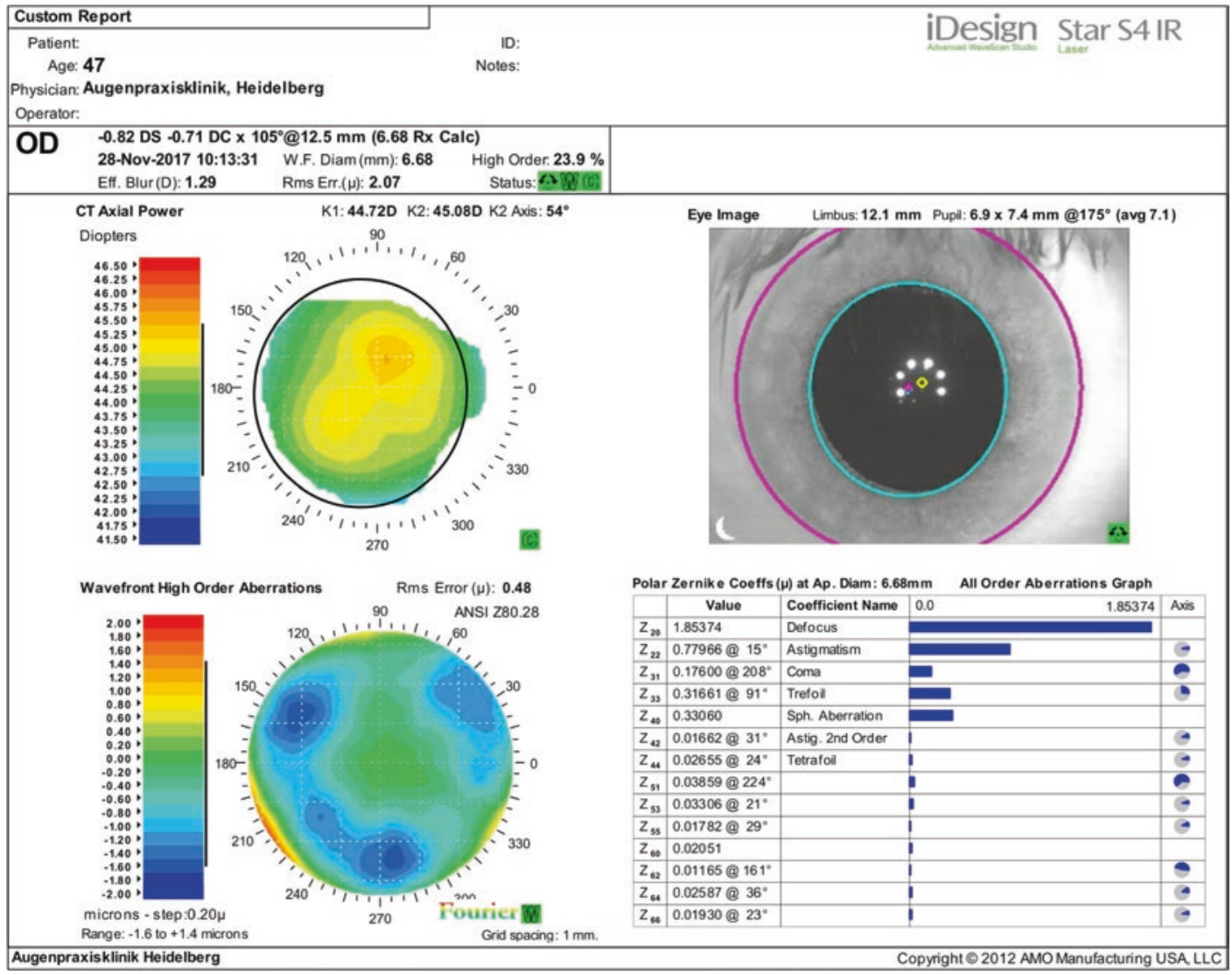

Fig. 18.11 Ocular aberrations measurement of right eye from a healthy volunteer with a commercially available aberrometer (iDesign) 
Heidelberg (Augenpraxisklinik Heidelberg). The pupil was dilated by the physicians for aberration measurements.

\subsubsection{Zemax Simulation}

The Zemax simulations were carried out to characterize the effect of aberration compensation using phase plates. One example is demonstrated in this section to evaluate the efficacy of phase plate in correcting the ocular aberrations. The Zernike coefficients from the iDesign measurement (Fig. 18.11, table) were used to simulate the wavefront map in Zemax. The piston, tilt and defocus are avoided for the simulation study since the piston and tilt correspond to lateral displacements of the image rather than the image degradation. Also, the defocus can be adjusted manually with the SPECTRALIS. The wavefront map simulated in Zemax is shown in Fig. 18.12 and is compared to the aberrometer measurement. The RMS error value and the wavefront map from Zemax simulation match with the aberrometer measurement.

Figure 18.13 shows the corresponding PSF and MTF of the eye before and after aberration compensation. The PSF and MTF clearly show that the aberrations severely impact the retinal imaging quality, and there will be a great improvement if these aberrations are compensated.

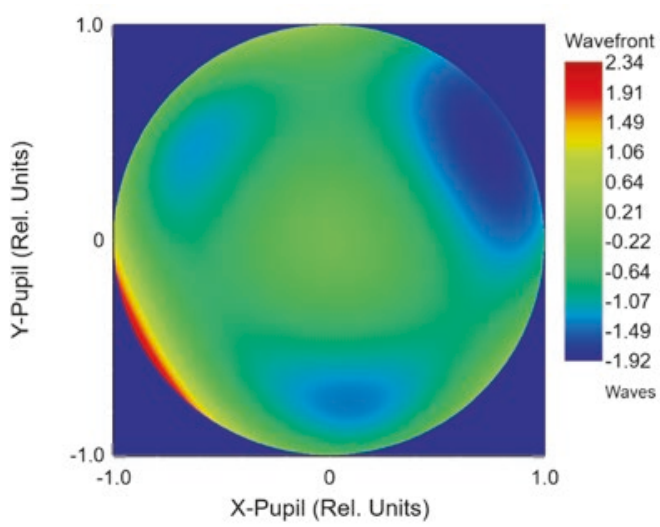

\subsubsection{Phase Plate}

A phase plate is a pre-compensation unit to correct higher-order aberrations in the human eye for retinal imaging. Diffraction-limited imaging can be achieved with phase plates by compensating the aberrations of the optical system and the human eye, providing a significant improvement in contrast of retinal images. With contrast improvement, more structural and morphological information can be retrieved from the retina. The phase plates can be manufactured using the Zernike coefficients of the measured ocular aberrations (Fig. 18.11, table), as the phase plate is the inverse wavefront of the measured aberrations. This inverse wavefront will make the aberrated wavefront flat and this will improve the contrast of a scanning laser ophthalmoscope. Figure 18.14 is an example showing how the phase plate can compensate and results in a flat wavefront.

\subsubsection{Mask Structured Ion Exchange Technique}

The mask structured ion exchange (MSI) technique is used to produce the phase plates. The mask structured silver-sodium ion exchange in glass (MSI) is a powerful tool for the realization of high precision refractive micro-optical components. A planar glass substrate is covered with a titanium layer on both sides. One side

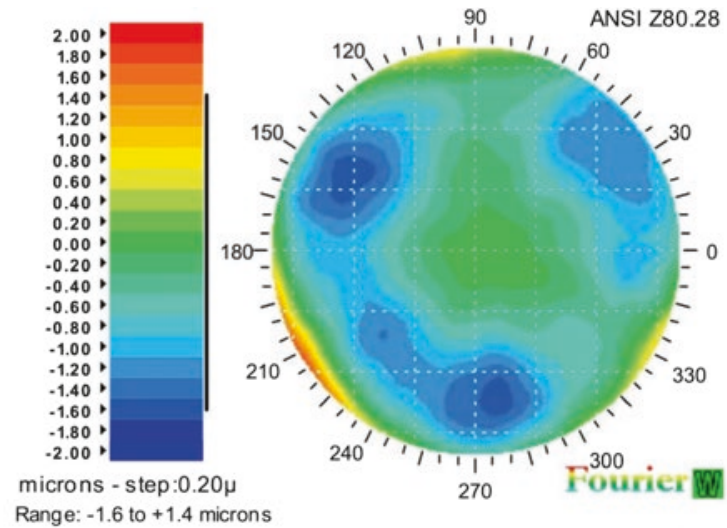

Fig. 18.12 Zemax simulation of wavefront map with higher-order aberrations without defocus and astigmatism (left image). Wavefront map of higher-order aberrations from aberrometer (right image). RMS $=0.48 \mu \mathrm{m}$ 

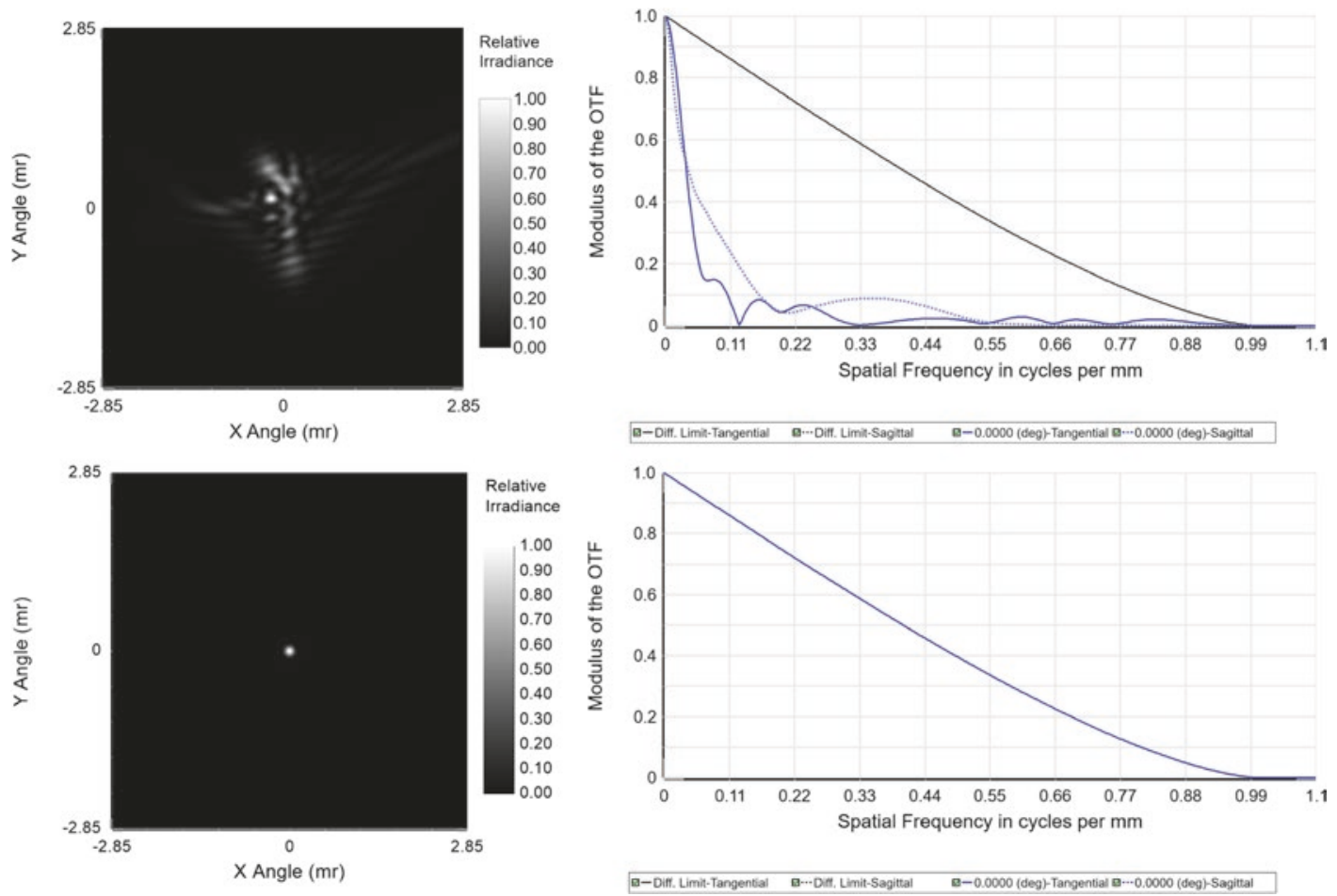

Fig. 18.13 PSF and MTF simulation in Zemax. PSF (top-left) and MTF (top-right) of aberrated eye. PSF (bottom-left) and MTF (bottom-right) after compensating the ocular aberrations
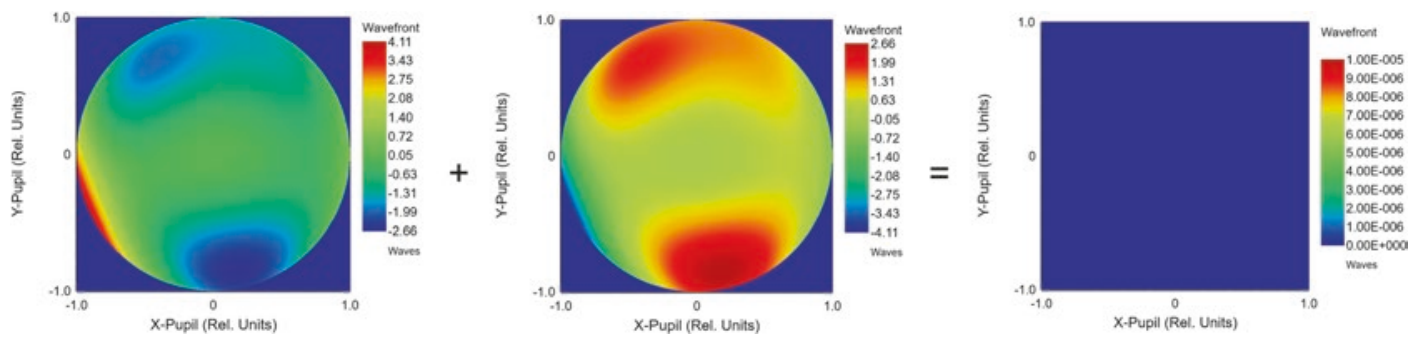

Fig. 18.14 Wavefront compensation with phase plate. Wavefront map of the aberrated eye (left image), wavefront map of the phase plate (middle image) and the resulting wavefront map (right image)

is structured by a photolithographic process to attain well-refined apertures for the ion migration into the glass material and the other side retains unstructured. The diffusion process takes place in a melt of $\mathrm{AgNO}_{3}$. The sodium ions are exchanged by silver ions from the melt (Fig. 18.15, left image). For the field assisted process (Fig. 18.15, right image) an additional electric field is applied between the silver salt melt (anode) and the bottom of the glass (cathode), which affects a current of silver ions into the glass [11].

\subsubsection{Phase Plate Specifications}

The customized phase plates for each individual were manufactured by Smart Microoptical Solutions, Germany. The size of the aberration compensation zone or active zone in each plate is $8 \mathrm{~mm}$ in diameter, and the phase plates are $2 \mathrm{~mm}$ thick (Fig. 18.16, left image). The phase plate can be easily fitted into SPECTRALIS with a phase plate holder (Fig. 18.16, right image). The length of the extension holder is about $55 \mathrm{~mm}$, and the 

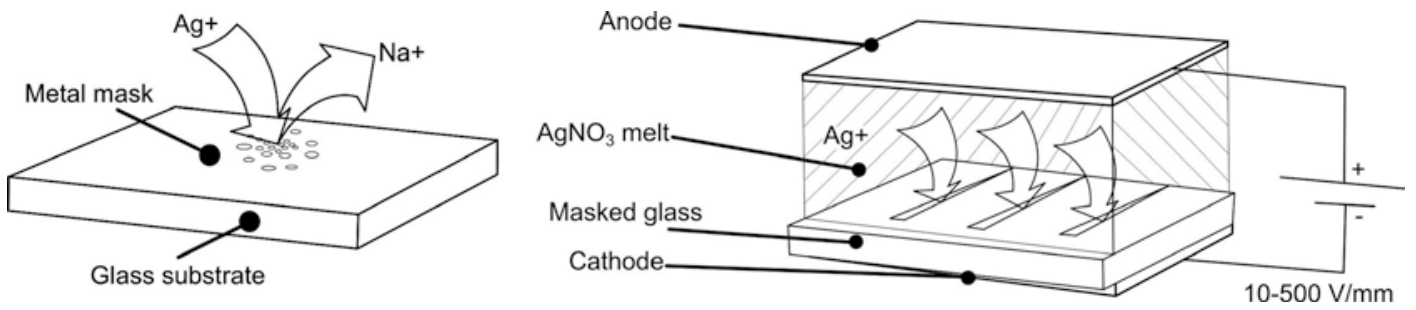

Fig. 18.15 Mask structured ion exchange technique. A thermal diffusion: exchange of $\mathrm{Na}^{+}$by $\mathrm{Ag}^{+}$ions (left image), field assisted process: $\mathrm{Ag}^{+}$ion current (right image) (adapted by permission from SPIE: [11])

Fig. 18.16 Photograph of phase plate (left image), and the photograph of SPECTRALIS with phase plate (right image)
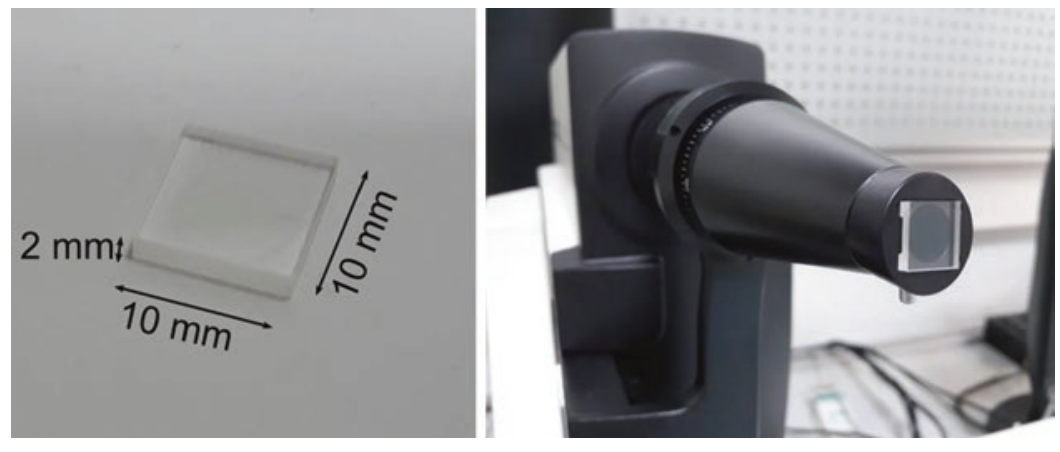

phase plates were positioned at the scan pupil of the scanning laser to compensate the aberrations. As a result, with the inclusion of phase plates in combination with a high-magnification objective lens, the aberrations can be compensated for high-contrast retinal imaging.

\subsubsection{Retinal Imaging with Phase Plates-Experimental Results}

In vivo measurements from healthy volunteers were performed in dilated and undilated eyes since the pupil size affects the image quality. It is expected that phase plates will increase the image quality in larger pupil size. However, with a large pupil size, the aberrations are higher compared to smaller pupil size and these aberrations have to be eliminated and diffraction should dominate to improve the optical quality. For pupil size smaller than $3 \mathrm{~mm}$, the ocular aberrations of the eye in healthy subjects are minimal and therefore, the retinal imaging of photoreceptors/microvasculatures with phase plates might not be immensely improved compared to the ret- inal imaging without phase plates. In this study, the efficacy of the phase plate on different pupil size has been evaluated. For retinal imaging in the dilated pupil, the subject's eyes were dilated with the pupil dilator at eye clinics in Heidelberg, and no other drugs were administered to prevent accommodation.

With SPECTRALIS, the NIR $(810 \mathrm{~nm})$ laser can be used for photoreceptor imaging and the green laser $(518 \mathrm{~nm})$ can be used for microvasculature imaging. Also, different layers of the retina can be visualized with the high-magnification objective lens by adjusting the focus. However, in this study, we focused on cone photoreceptors to determine the efficacy of phase plates in retinal imaging with NIR.

Images visualizing cone photoreceptors in healthy volunteers were acquired with $8^{\circ}$ and $4^{\circ}$ FOV. A significant improvement in image quality with phase plates compared to images without phase plates was noticed in both dilated (Fig. 18.17) and undilated eyes (Fig. 18.18). In Fig. 18.17, top left image visualizes the retina in a dilated eye with $4^{\circ} \mathrm{FOV}$ with cone photoreceptors clearly visible. The contrast improvement 

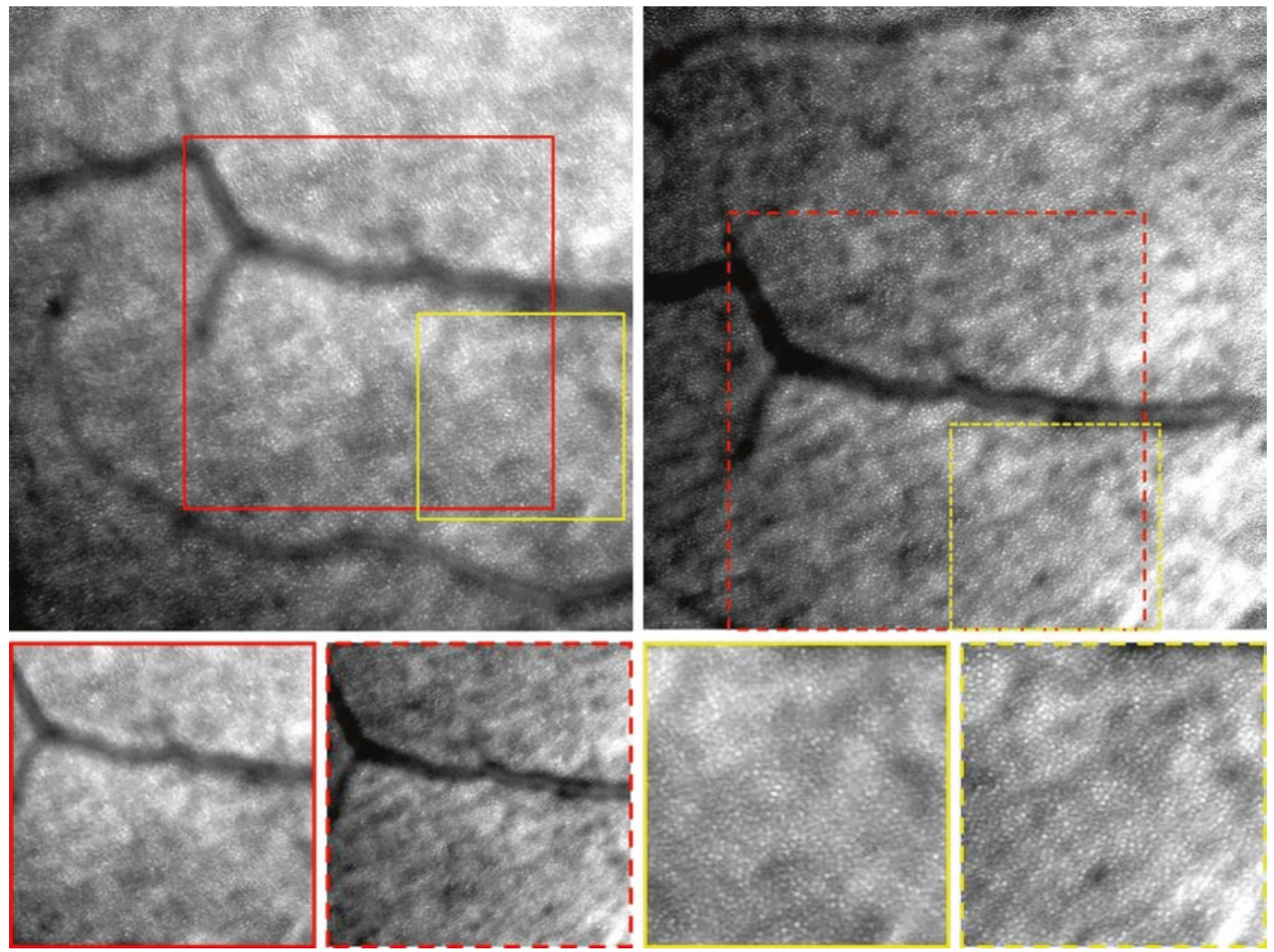

Fig. 18.17 High-magnification retinal images in a dilated eye without (top-left) and with (top-right) phase plate. Comparison of retinal image quality without (solid line boxes) and with (dotted line boxes) phase plate

was also noticed with the implementation of phase plates (Fig. 18.17, right image). Likewise, the improvement in contrast and visualization of cone photoreceptors was noticed in the undilated pupil as well (Fig. 18.18). The comparison of retinal image quality without phase plate (Figs. 18.17 and 18.18, solid line boxes) and with phase plate (Figs. 18.17 and 18.18, dotted line boxes) clearly supports that the improvement in contrast from the cones photoreceptors with the implementation of the phase plate. This proves that the phase plates will facilitate examining the individuals suffering from higher-order aberrations.

The cone photoreceptors with the largest FOV $\left(8^{\circ}\right)$ was resolved in this study; however, with large FOV degradation in the corners of the image was noticed. Also, the foveal cone photoreceptors could not be optically resolved with larger FOV. This could be due to the isoplanatic angle of the eye, which is in the order of $3-4^{\circ}$, and the sampling density might not be sufficient to resolve small structures. The $4^{\circ} \mathrm{FOV}$ in our test device was only a digital zoom, and this does not provide more physical information or better resolution, and the retinal structures cannot be better resolved. But the $4^{\circ}$ FOV could be used to better position the focal plane at the retina for photoreceptor imaging and to eliminate the image degradation in the corners.

A widefield photoreceptor imaging can be achieved by montaging. The retinal images can be acquired from nine different locations using the fixation target and then can be stitched together for a wide FOV. With the stitched images the FOV of $15^{\circ}$ can be achieved with the SPECTRALIS. However, due to the size of photoreceptors, the visibility of small details cannot be fully appreciated with stitching (Fig. 18.19) and manual zoom in of the images is required. 

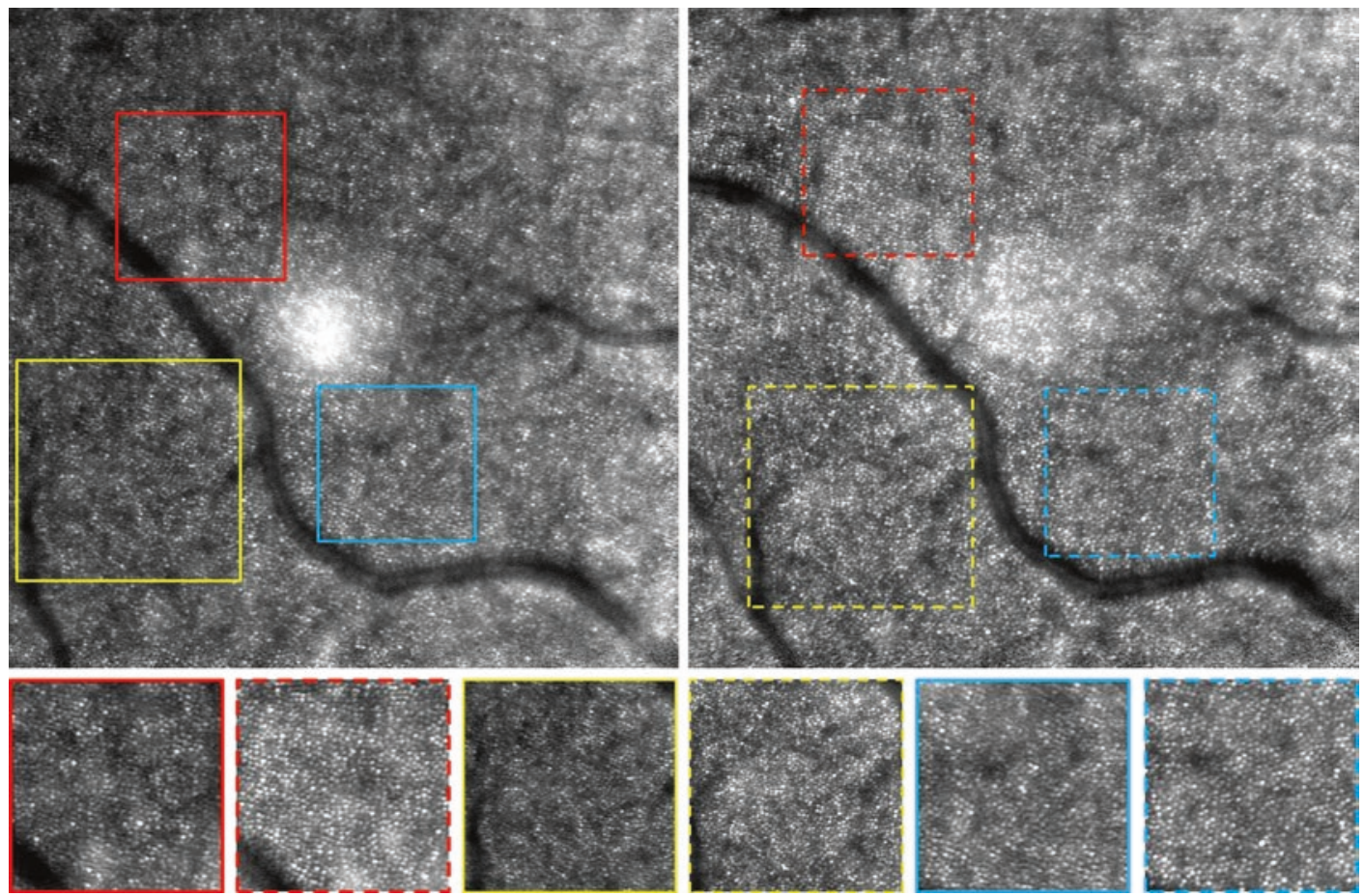

Fig. 18.18 High-magnification retinal images of an undilated eye without (top-left) and with (top-right) phase plate. Comparison of retinal image quality without (solid line boxes) and with (dotted line boxes) phase plate
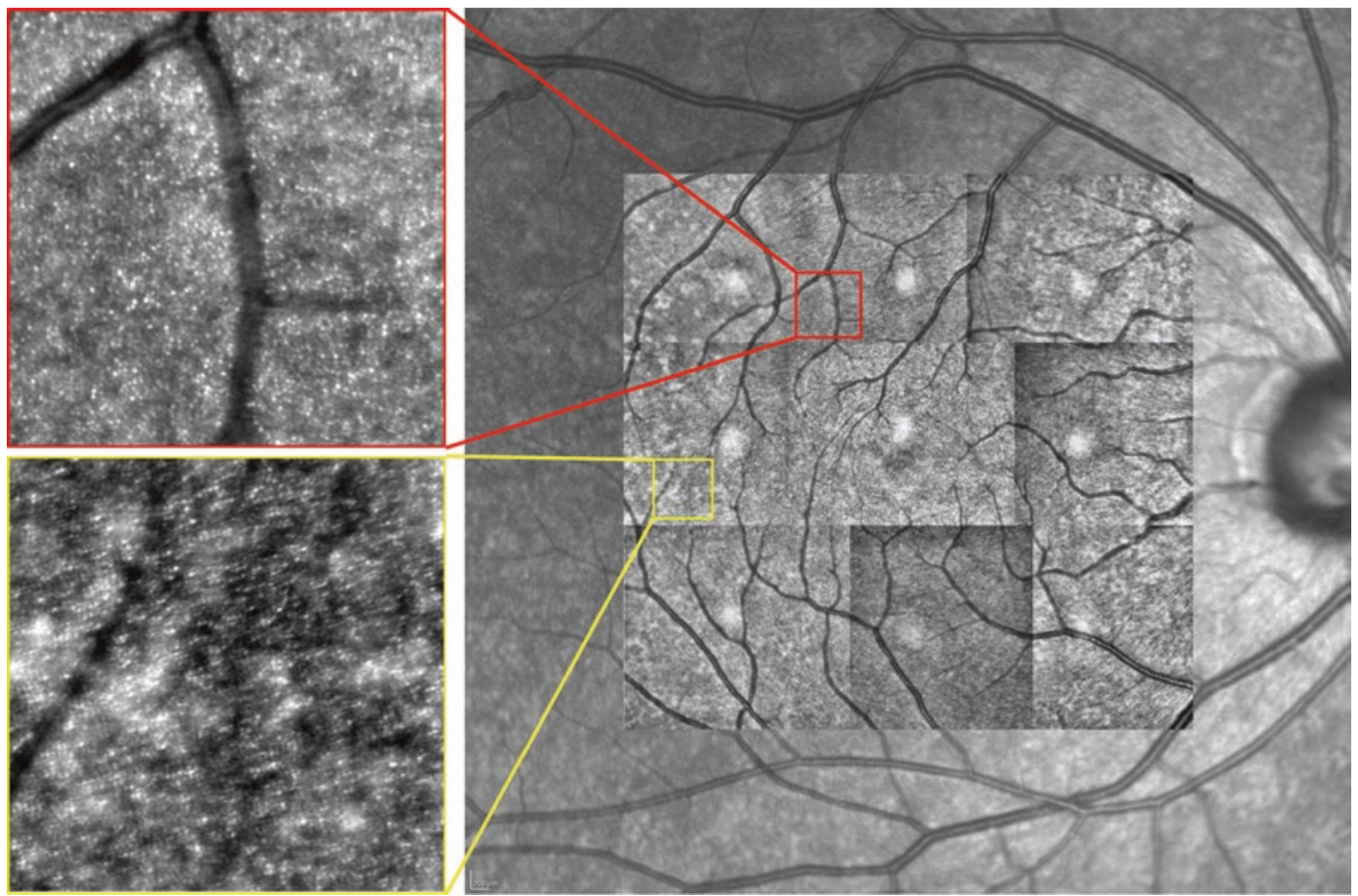

Fig. 18.19 $8^{\circ} \mathrm{FOV}$ stitched images in $30^{\circ} \mathrm{FOV}$ retinal image and zoom-in images of $8^{\circ} \mathrm{FOV}$ (red and yellow box) 

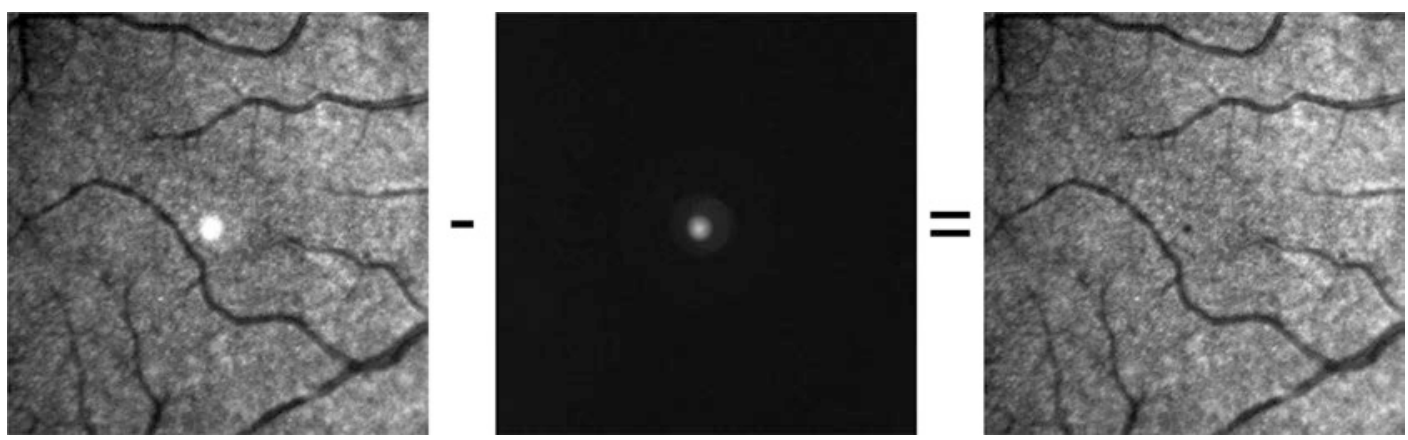

Fig. 18.20 Retinal image (left image), background image (middle image), and the retinal image after background subtraction (right image)

Currently, the retinal images acquired with the high-magnification objective lens show a bright white spot in the center of the image. The bright white spot is a result of the reflection from the lens itself and the reflection also has an effect on the pupil size of the acquired eye. The laser beam diameter of the high-magnification objective lens is $6 \mathrm{~mm}$ and therefore for smaller pupil sizes the reflection from the lens was visible on the images. The reflection was not noticed on the retinal image from the dilated eyes (see Figs. 18.17 and 18.18). These reflections on the acquired images can be eliminated by subtracting the acquired retinal image from the background image (Fig. 18.20).

\subsection{Conclusion and Discussion}

The study confirmed that phase plates can compensate the ocular aberrations and improve the retinal image quality. The lower-order aberrations such as defocus and astigmatism can be compensated using sphero-cylindrical lenses. These lenses are called astigmatic lenses and included with the high-magnification objective lens. In this study, the phase plates were tested in healthy volunteers compensating the lower and higher-order aberrations together. With the help of wavefront technology, the optical aberrations in the human eye can be precisely measured and help in producing the phase plates.

Compared to adaptive optics systems, the phase plates are much easier to adapt to existing clinical ophthalmoscopes and are less expensive.
Significant improvements in retinal image quality can be achieved by introducing this technology to the cSLO. Improved diagnosis and detection of retinal diseases at a cellular level will be clinically relevant and will add value for patients as well as for research.

Although improvement in retinal image quality is noticed, this study is in a preliminary stage. Some improvement is further needed to implement the phase plates in clinical routine. Currently, the customized phase plates have been tested only on healthy volunteers with minimum aberrations, and as a next step, the phase plates need to be tested on individuals suffering from higherorder aberrations. In our experimental setup, the wavefront sensor is not included and commercial aberrometer has been used to measure the ocular aberrations to produce phase plates. It will be a great improvement if a Hartmann-Shack sensor has been employed to the system to measure the aberrations. This will allow us to measure both the system and ocular aberrations together and no additional device needed for ocular aberration measurements. Also, having a wavefront sensor in our system could improve the optimum degree of compensation because the positioning of phase plates is crucial for retinal imaging. Proper positioning of phase plates is a key factor to achieve the optimum degree of compensation, as some misalignments could reduce the performance of the phase plate. Therefore, implementing a wavefront sensor could guide the photographers to position the patient's eye for effective optical compensation. 
With respect to cSLO, the phase plates could be implemented in other retinal imaging technologies as well. The phase plates with OCT could improve the lateral resolution compensating the aberrations, and also could be used for two-photon ophthalmoscopy for diffraction-limited imaging [7]. The phase plates can also be used for selective retinal therapy (SRT) since the laser can be focused precisely on the retina by compensating the aberrations of the human eye. In future, combining the SRT with two-photon ophthalmoscopy and phase plates can advance the SRT treatment, since with the two-photon principle the laser can be focused more precisely on the retinal pigment epithelium (RPE) without radiating other retinal tissue layers. Also, the RPE can be visualized with two-photon retinal imaging, and these images could be used as a reference image for the SRT procedure. In conclusion, the phase plates show significant improvements in retinal image quality with cSLO and are promising for improved diagnosis and detection of retinal diseases for subjects affected by higher-order optical aberrations.

\section{References}

1. Marcos S, Werner J, Burns S, Merigan W, Artal P, Atchison D, Hampson K, Legras R, Lundstrom L, Yoon G, Carroll J, Choi S, Doble N, Dubis A, Dubra A, Elsner A, Jonnal R, Miller D, Paques M, Smithson H, Young L, Zhang Y, Campbell M, Hunter J, Metha A, Palczewska G, Schallek J, Sincich L. Vision sci- ence and adaptive optics, the state of the field. Vis Res. 2017;132:3-33.

2. Liang J, Williams DR, Miller DT. Supernormal vision and high-resolution retinal imaging through adaptive optics. J Opt Soc Am A. 1997;14:2884-289.

3. Porter J, Queener H, Lin J, Thorn K, Awwal A, editors. Adaptive optics for vision science: principles, practices, design, and applications. Hoboken, NJ: John Wiley \& Sons; 2006.

4. Merino D, Loza-Alvarez P. Adaptive optics scanning laser ophthalmoscope imaging: technology update. Clin Ophthalmol. 2016;10:743-55.

5. LaRocca F, Dhalla AH, Kelly MP, Farsiu S, Izatt JA. Optimization of confocal scanning laser ophthalmoscope design. J Biomed Opt. 2013;18(7):076015.

6. Zhang H. A study of aberrations in the human eye by zernike phase plate precompensation and finite element modeling methods. Dissertation, University of Heidelberg. 2007.

7. Bille JF, Agopov M, Alvarez-Diez C, Han M, Koralinova N, von Pape U, Schiazza O, Schwingel M, Zhang H, Mueller F. Compact adaptive optics system for multiphoton fundus imaging. J Mod Opt. 2008;55:749-58.

8. Resan M, Vukosavljević M, Milivojević M. Wavefront aberrations. In Ophthalmolgoy Shimon Rumelt. 2012. https://www.intechopen.com/books/ advances-in-ophthalmology/wavefront-aberrations.

9. American National Standard for Ophthalmics (ANSI Z80.28-2004) Methods for reporting optical aberrations of eyes.

10. Liang J, Grimm B, Goelz S, Bille JF. Objective measurement of wave aberrations of the human eye with the use of a Hartmann-Shack wave-front sensor. J Opt Soc Am A. 1994;11(7):1949-57.

11. Baehr J, Brenner KH. Applications and potential of the mask structured ion exchange technique (MSI) in micro-optics. In: Proc SPIE, vol. 5177; 2003. p. 121-32.

Open Access This chapter is licensed under the terms of the Creative Commons Attribution 4.0 International License (http://creativecommons.org/licenses/by/4.0/), which permits use, sharing, adaptation, distribution and reproduction in any medium or format, as long as you give appropriate credit to the original author(s) and the source, provide a link to the Creative Commons license and indicate if changes were made.

The images or other third party material in this chapter are included in the chapter's Creative Commons license, unless indicated otherwise in a credit line to the material. If material is not included in the chapter's Creative Commons license and your intended use is not permitted by statutory regulation or exceeds the permitted use, you will need to obtain permission directly from the copyright holder. 